\title{
Causes of 30-day readmission after neurosurgery of the spine
}

\author{
Michael D. Cusimano, MD, PhD, ${ }^{1,2}$ Iryna Pshonyak, BSc, ${ }^{1}$ Michael Y. Lee, ${ }^{1}$ and Gabriela Ilie, PhD1 \\ 1'Division of Neurosurgery, Keenan Research Centre and Li Ka Shing Knowledge Institute, St. Michael's Hospital; and \\ 2Dalla Lana School of Public Health and Department of Surgery, University of Toronto, Ontario, Canada
}

\begin{abstract}
OBJECTIVE Thirty-day readmission has been cited as an important indicator of the quality of care in several fields of medicine. The aim of this systematic review was to examine rate of readmission and factors relevant to readmission after neurosurgery of the spine.

METHODS The authors carried out a systematic review using several databases, searches of cited reference lists, and a manual search of the JNS Publishing Group journals (Journal of Neurosurgery; Journal of Neurosurgery: Spine; Journal of Neurosurgery: Pediatrics; and Neurosurgical Focus), Neurosurgery, Acta Neurochirurgica, and Canadian Journal of Neurological Sciences. A quality review was performed using STROBE (Strengthening the Reporting of Observational Studies in Epidemiology) criteria and reported according to the PRISMA (Preferred Reporting Items for Systematic Reviews and Meta-Analyses) guidelines.
\end{abstract}

RESULTS A systematic review of 1136 records published between 1947 and 2014 revealed 31 potentially eligible studies, and 5 studies met inclusion criteria for content and quality. Readmission rates varied from $2.54 \%$ to $14.7 \%$. Sequelae that could be traced back to complications that arose during neurosurgery of the spine were a prime reason for readmission after discharge. Increasing age, poor physical status, and comorbid illnesses were also important risk factors for 30-day readmission.

CONCLUSIONS Readmission rates have predictable factors that can be addressed. Strategies to reduce readmission that relate to patient-centered factors, complication avoidance during neurosurgery, standardization with system-wide protocols, and moving toward a culture of nonpunitive system-wide error and "near miss" investigations and quality improvement are discussed.

http://thejns.org/doi/abs/10.3171/2015.4.SPINE15445

KEY WORDS readmission; spine; systematic review; neurosurgery; outcomes

$\mathrm{I}$ $\mathrm{N}$ the last 20 years, the burdens of readmission on the health care system, hospitals, patients, and insurers have become apparent. Estimates are that Medicare spends over $\$ 15$ billion on readmission-related expenditures yearly. ${ }^{21,32}$ Readmission can be influenced by the quality of in-hospital care as well as follow-up care in the community, among other factors. ${ }^{17}$ Compared with the US, in Canada, the interest in readmission is still growing. A recent report on readmissions by Health Canada found the current 28-day readmission rate for acute myocardial infarction to be concerning. ${ }^{17}$ Rates of readmission were $6.2 \%$ in 2003-2004 to $2005-2006,4.4 \%$ in $2007-2008$ to 2009-2010, and 4.1\% for 2008-2009 to 2010-2011. Ac- cording to this report, readmission rates are considered important factors for measuring the quality and efficiency of acute care. The report also called the need for reducing readmission rates a public health priority. Multifaceted interventions are now provided by health care professionals, which have been shown to be effective in readmission rate reductions.${ }^{17}$ With multiple programs concerned with improvement of quality of care and readmission being viewed as an indicator of hospital performance, readmission after discharge has become a principal target for cost reduction..$^{21}$

In the United States, 30-day readmission after discharge is an important indicator of health care quality in

ABBREVIATIONS ASA = American Society of Anesthesiologists; $C P T=$ Current Procedural Terminology; DVT = deep vein thrombosis; LOS = length of stay; $P C I=$ percutaneous coronary intervention; PE = pulmonary embolism; PRISMA = Preferred Reporting Items for Systematic Reviews and Meta-Analyses; SSI = surgical site infection; $\mathrm{UTI}=$ urinary tract infection.

SUBMITTED January 2, 2015. ACCEPTED April 15, 2015.

INCLUDE WHEN CITING Published online October 9, 2015; DOI: 10.3171/2015.4.SPINE15445. 
cardiac, pulmonary, and general medicine..$^{32}$ In contrast to these specialty areas, in neurosurgery, the connection between the rates of readmission and performance level is not as clear and may be considered an important element of good quality care. ${ }^{17,32}$ Given that neurosurgery patients can decline rapidly in clinical course, having vague warning signs and outcomes that quite often are unpredictable, early intervention can prevent complications, serious morbidities, and disease progression. Hence, it has been argued that high rates of 30-day readmission in neurosurgery are a sign of prudent care that may ultimately indicate better outcomes and better quality of care rather than the contrary, as is seen in other medical specialties. ${ }^{32}$

Studies reporting rates and the significance of early readmission after neurosurgery in general and after neurosurgery of the cervical, thoracic, and lumbar spine are rare. Understanding all of these reports is necessary to understand why readmission occurs in neurosurgery of the spine, and if there are any factors that lend themselves to the development of strategies to prevent their occurrence.

In this study, our aim was 3-fold. First, we aimed to report available information on 30-day readmission rates after neurosurgery of the spine and examine commonalities and disparities presented in literature. Our second goal was to identify the factors that are associated with readmission and to identify any at-risk populations. Finally, we aimed to discuss potential strategies that might ameliorate any of the identified risk factors for readmission.

\section{Methods}

A search of the English-language literature was conducted using both electronic and hard-copy resources. A total of 1131 records were obtained using Medline (1946-June 2014) and Embase Classic (1947-June 2014); 5 additional articles were found by manual searches of the JNS Publishing Group journals (Journal of Neurosurgery; Journal of Neurosurgery: Spine; Journal of Neurosurgery: Pediatrics; and Neurosurgical Focus), Neurosurgery, Acta Neurochirurgica, Canadian Journal of Neurological Sciences, and the cited references of the selected articles as well as using the search cited features of PubMed. The search words used to obtain these articles included disk disease or spinal disease, spinal stenosis (exploded) (terms combined using an OR statement), followed by surgical specialties (exploded), surgery or surgical, neurosurgery (exploded), neurosurgical procedures (exploded), laminectomy, discectomy, foraminotomy, denervation, microvascular decompression surgery, nerve transfer (terms combined using an OR statement). Finally, the search used terms patient readmission (exploded) and reoperation (exploded) and readmi*, admi*, rehospitaliz*, hospitaliz*. Articles were limited to observational studies; case series; case controls; cross-sectional surveys; randomized controlled trials; and retrospective, prospective, and follow-up studies assessing only human cohorts. Studies without primary data (commentary, letters, reviews, editorials, and methods papers), case studies, and case reports were excluded.

Studies that explored readmission after neurosurgery for spinal stenosis or disk disease were included in the review. We also searched for and included the following procedures: cervical decompression (anterior and posterior approach), lumbar discectomy, and lumbar decompression.

Studies were included if they reported the number of patients readmitted to the hospital after neurosurgery (including demographics and reasons for readmission) and compared it to patients who were not readmitted, to a total population, or to a population of readmitted patients after a different procedure. Articles that reported readmission as part of a composite outcome, complications, reoperation, mortality, or morbidity were excluded. There were no limitations to the population size, age, or sex.

Of the total articles reviewed, 31 articles were deemed as potentially eligible. The remaining 1105 articles were excluded based on the above criteria. Further screening of the potentially eligible articles through full-text examination resulted in the exclusion of 26 articles and the selection of only 5 of the remaining articles for final inclusion. A PRISMA (Preferred Reporting Items for Systematic Reviews and Meta-Analyses) flowchart, shown in Fig. 1, was created to illustrate the number of articles found at each stage of data acquisition and the number of articles that were excluded at each stage. Two of the authors (I.P. and M.L.) independently evaluated the quality of the articles in the search and extracted data using data abstraction forms. The STROBE (Strengthening the Reporting of Observational Studies in Epidemiology) criteria for quality assessment were applied to evaluate each article on study quality and external and internal validity. ${ }^{37}$ Agreement between the 2 raters was very high (Cohen's kappa $=0.85$ ). Results are reported according to the PRISMA guidelines. $^{22}$

\section{Results}

All of the selected studies assessed readmission rates and compared readmitted and nonreadmitted patients ${ }^{21,24,30}$ or the readmitted and overall patient population ${ }^{8,19}$ and identified vulnerable populations and evaluated risk factors for readmission after neurosurgery of the spine. Investigated procedures included lumbar decompression, discectomy, laminectomy, and fusion ${ }^{8,21,30}$ and anterior cervical discectomy and fusion and corpectomy. ${ }^{24}$ One of the studies did not report which specific neurosurgical procedure was performed..$^{19}$ Two studies identified complications and their prevalence after surgical procedures. ${ }^{21,24}$

Table 1 displays study characteristics. Of the selected articles, $4^{8,21,24,30}$ were conducted in the United States and 1 was conducted in Sweden..$^{19}$ All of the studies were retrospective (longitudinal) cohorts and retrieved multiinstitutional data from time periods ranging from 1987 to 2012. $8,19,21,24,30$ The total patient population in each study varied from 2320 to 35,152 . The methods of patient selection differed throughout the studies. Most of the studies (4 of 5) used ICD-9 or Current Procedural Terminology (CPT) codes to identify the appropriate neurosurgical diagnoses and/or procedures..$^{19,21,24,30}$ Nearly all of the cohorts had readmission as the primary study outcome (4 of 5 studies), ${ }_{19,21,24,30}$ with 1 cohort reporting rehospitalization. ${ }^{8}$ Thirty-day readmission rates ranged from $2.54 \%$ 


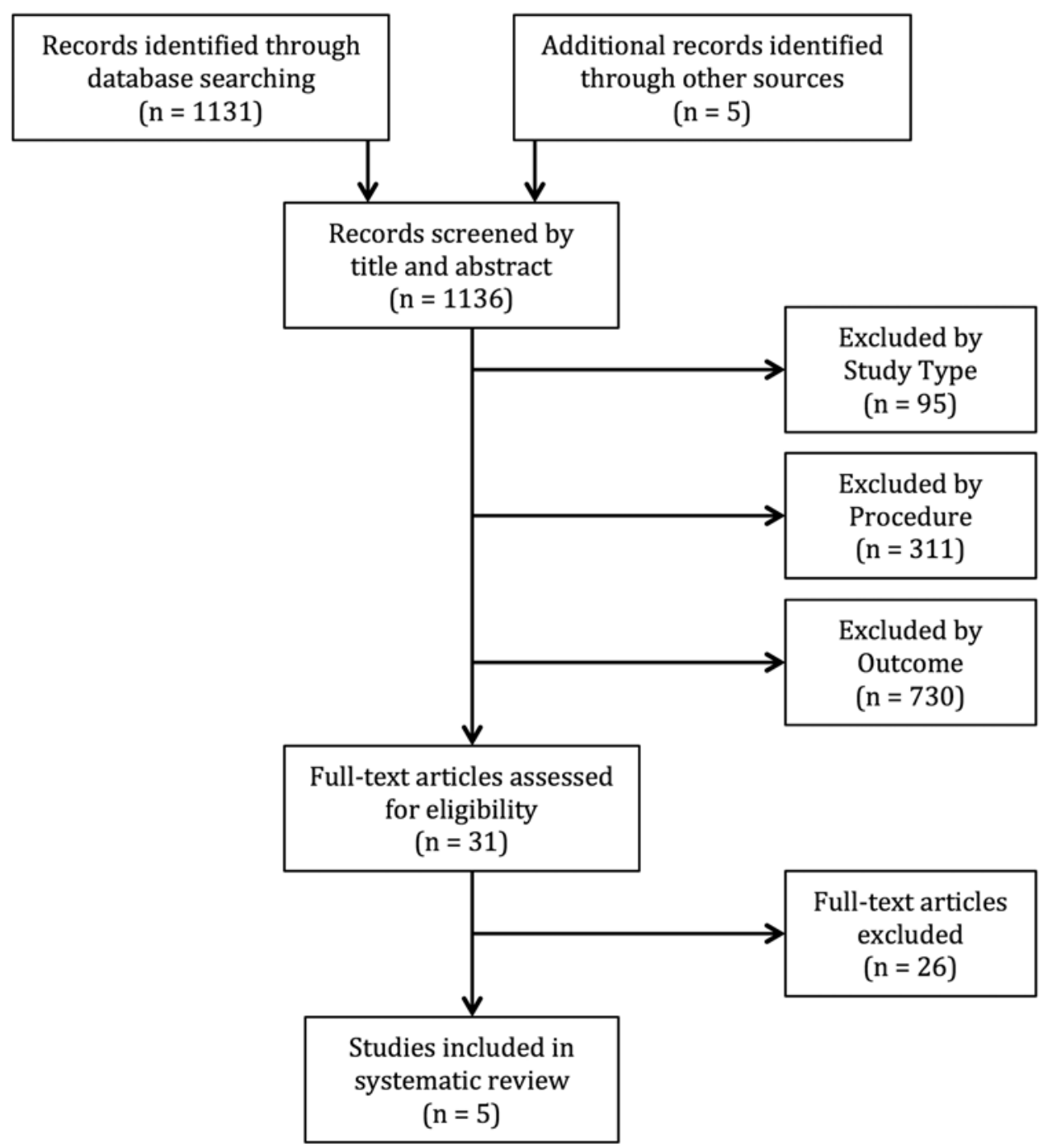

FIG. 1. PRISMA diagram showing the flow of information through the different phases of the systematic review.

to $14.7 \%$. Three of the 5 selected studies ${ }^{21,24,30}$ compared the readmitted to nonreadmitted patients while the remaining 2 studies $^{8,19}$ used the overall patient population as control.

Analytical methods used to examine predictors of readmission included chi-square tests, ${ }^{8,21,24,30}$ linear regression, ${ }^{8}$ t-tests, ${ }^{8,30}$ and analyses of variance. ${ }^{8,30}$ Four of the 5 studies used the multivariate logistic regression model to identify the risk factors for readmission..$^{8,21,24,30}$ Of the 5, 3 studies further examined factors associated with 30-day readmission, using univariate analysis. ${ }^{21,24,30}$ One of the 5 studies used the Cox proportional hazards model to determine the effect of readmission on survival. ${ }^{19}$ Statistical significance was determined in all studies using a $p$ value of 0.05 or less. None of the studies reported power analyses to determine if their study had a sufficient sample size to detect an effect, if an effect was existent.

There was consistency across the studies in the variables age and length of stay (LOS) presented in the analytical models (Table 2); however, consistency was lacking in sex and race. In all studies, age reliably distinguished be- tween the compared groups (either readmitted vs nonreadmitted or readmitted vs overall)..$^{8,19,21,24,30}$ Overall, readmission rates after all procedures and age were positively correlated..$^{8,21,24,30}$ One study found a negative correlation between these 2 factors among ages 50-69 years. ${ }^{19}$ Longer LOS (at index admission) was found to be a significant variable in lumbar spine surgery, as compared with overall $^{19}$ or nonreadmitted ${ }^{30}$ patients. In 3 of the 5 studies, ${ }^{8,19,30}$ sex was a significant predictor of condition (readmitted vs overall and nonreadmitted), with females returning more frequently than males..$^{8,19,30}$ However, results were inconclusive, as Jansson et al. ${ }^{19}$ found males to return more frequently while Deyo et al. ${ }^{8}$ and Pugely et al. ${ }^{30}$ reported the opposite. In the remaining 2 studies, ${ }^{21,24}$ sex was not found to be significant. Being of African American race was among the reported risk factors. ${ }^{30}$ Three other studies reported race to be not significant. ${ }^{8,21,24}$

Higher comorbidity score was found to be a significant predictor in 1 study that reported the variable. ${ }^{8}$ Among the comorbidities listed, having diabetes, ${ }^{21,24,30}$ hypertension, ${ }^{21,24,30}$ and previous percutaneous coronary interven- 


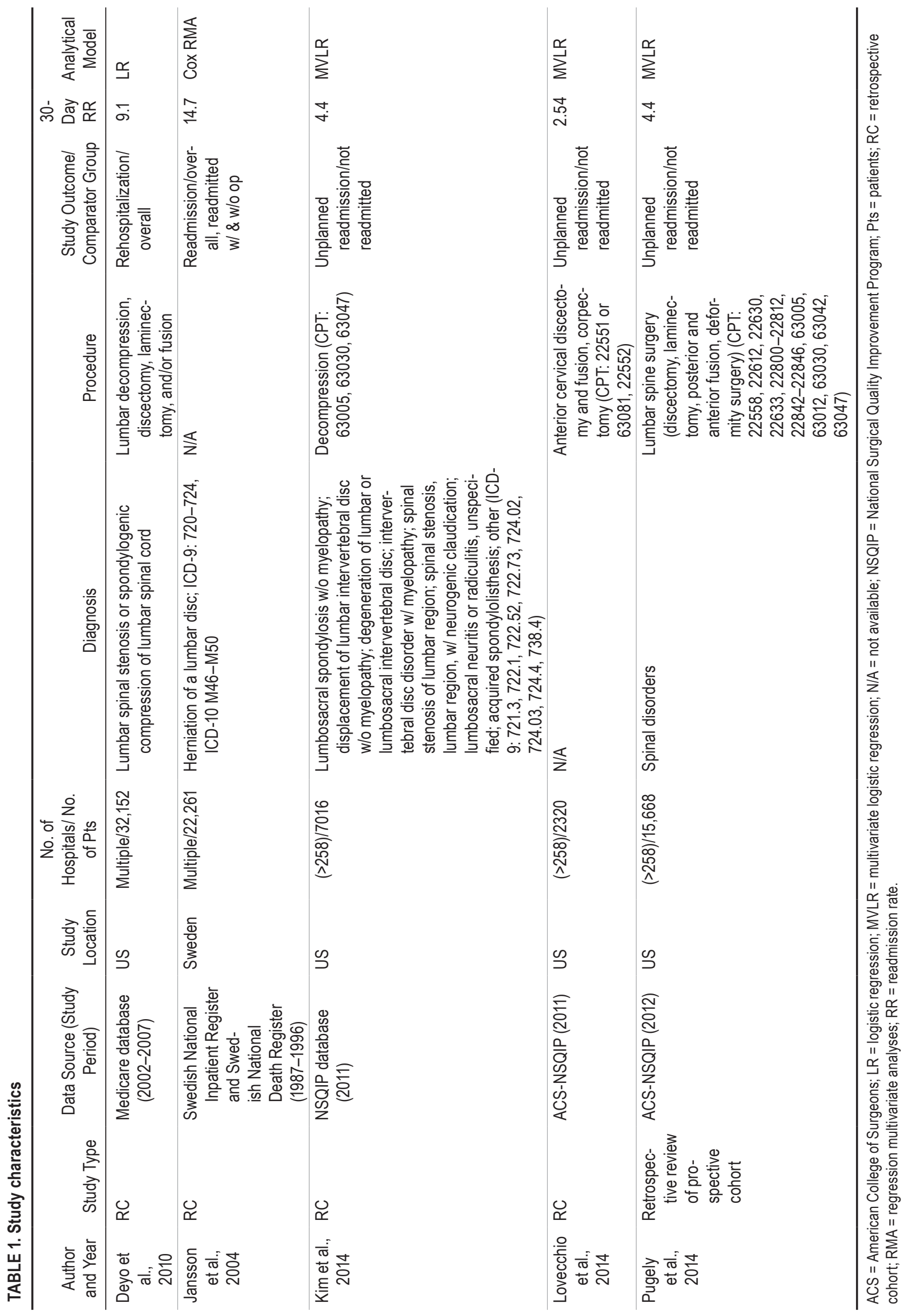


Table 2. Demographic and clinical contributions of patient characteristics (after univariate analyses)

\begin{tabular}{|c|c|c|c|c|c|}
\hline \multirow[b]{2}{*}{ Characteristic } & \multicolumn{5}{|c|}{ Study } \\
\hline & $\begin{array}{l}\text { Deyo et al., } \\
2010\end{array}$ & $\begin{array}{l}\text { Jansson et al., } \\
2004\end{array}$ & $\begin{array}{c}\text { Kim et al., } \\
2014\end{array}$ & $\begin{array}{l}\text { Lovecchio et al., } \\
2014\end{array}$ & $\begin{array}{c}\text { Pugely et al., } \\
2014\end{array}$ \\
\hline Age & S & S & $S$ & $S$ & S \\
\hline Sex & $S$ & $S$ & NS & NS & $S$ \\
\hline Race & NS & 0 & NS & NS & $S$ \\
\hline Diagnoses & NS & 0 & 0 & 0 & 0 \\
\hline Procedure & $S$ & 0 & $S$ & 0 & 0 \\
\hline Complexity of procedure & $S$ & 0 & $S$ & 0 & 0 \\
\hline LOS $^{*}$ & 0 & $S$ & 0 & 0 & $S$ \\
\hline Previous hospitalization & $S$ & S & 0 & 0 & 0 \\
\hline ASA Class & 0 & 0 & $S$ & $S$ & S \\
\hline $\mathrm{BMI}$ & 0 & 0 & NS & NS & $S$ \\
\hline Hematocrit & 0 & 0 & $S$ & 0 & $S$ \\
\hline Period & 0 & NS & 0 & 0 & 0 \\
\hline Comorbidity score & $S$ & 0 & 0 & 0 & 0 \\
\hline
\end{tabular}

$\mathrm{BMI}=$ body mass index; $\mathrm{NS}=$ not a statistically significant contributor; $\mathrm{O}=$ not assessed/considered in the model; $\mathrm{S}=$ statistically significant contributor.

* With reference to index admission.

tion $(\mathrm{PCI}) /$ cardiac surgery ${ }^{21,24}$ were positively correlated with readmission rates (Table 3). History of previous hospitalization, 8,19 higher American Society of Anesthesiologists (ASA) Physical Status Classification, ${ }^{21,24,30}$ longer operative time, ${ }^{21,24,30}$ history of previous hospitalization, ${ }^{8,19}$ and hospital admission for spinal symptoms ${ }^{19}$ were also among the identified risks. Other factors determined to be significant were expected primary payer ${ }^{30}$ and anemia. ${ }^{21}$

Kim et al. ${ }^{21}$ and Lovecchio et al. ${ }^{24}$ reported that complication rates after the procedures differ between readmitted and not readmitted populations. Deyo et al., Jansson et al. ${ }^{19}$ and Pugely et al. ${ }^{30}$ listed complications in terms of reasons for readmission but did not provide comparative analysis between the groups. Kim et al..$^{21}$ and Lovecchio et al. ${ }^{24}$ agree that surgical and wound complications, such as incisional and organ surgical site infections (SSIs), appear significantly more frequent in readmitted patients as compared with nonreadmitted..$^{21}$ Deyo et al. ${ }^{8}$ and Pugely et al. ${ }^{30}$ also reported surgical and wound complications as one of the main reasons for readmission.

Kim et al. ${ }^{21}$ and Lovecchio et al. ${ }^{24}$ reported on systemic infectious complications. Both authors argued that urinary tract infection (UTI) rates were significantly lower in the nonreadmitted group compared with readmitted. Blood transfusion was less common in nonreadmitted patients. ${ }^{21,24}$ Sepsis was more prevalent in the readmitted population ${ }^{21,30}$; however, after risk-adjusted multivariate logistic regression analysis this difference was not significant. In addition, in their report, Lovecchio et al. ${ }^{24}$ did not find sepsis rates to be statistically significant between the 2 populations. Finally, there was no consensus on whether mortality rates between the groups differed significantly.

Other medical complications were reported in several of the studies reviewed (Table 4). Pulmonary complications were reported as significantly lower for the nonreadmitted group. ${ }^{21,24}$ However, Kim et al. ${ }^{21}$ note that mechanical ventilation $>48$ hours, as one of the pulmonary complications, was statistically not significant. Deyo et al. ${ }^{8} \mathrm{Kim}$ et al. ${ }^{21}$ Lovecchio et al., ${ }^{24}$ and Pugely et al. ${ }^{30}$ reported cardiac complications as reasons for readmission. However, Lovecchio et al. ${ }^{24}$ found the rate of cardiac complications did not significantly differ between readmitted and nonreadmitted groups. No consistency regarding the rate of vascular complications in the compared groups was observed.

Venous complications such as deep vein thrombosis (DVT) and pulmonary embolism (PE) rates were consistently higher in the readmitted population. ${ }^{21,30}$ Lovecchio et al. ${ }^{24}$ did not find the difference significant. Renal complications were reported as insignificant. Stroke is frequently mentioned as a complication ${ }^{8,21,30}$ resulting in readmission; however, only Kim et al. ${ }^{21}$ reported stroke as significantly more prevalent in the readmitted population.

\section{Discussion}

Our study found that 30-day readmission rates varied from $2.54 \%$ to $14.7 \%$ after neurosurgical spine procedures. The wide variability is likely due to the heterogeneity of studies. Each reviewed article varied on inclusion and exclusion criteria, procedures and diagnoses, comparison groups, duration of study follow-up, sample size, geographic location, and analytical methods. Although focused on spinal procedures, each study included different sets of diagnostic codes, some using $\mathrm{CPT}^{21,24,30}$ and others using the ICD- $9^{8}$ classification codes, while Jansson et al. ${ }^{19}$ used Swedish classification. Clearly, the rate of readmission will differ based on the period of observation postdischarge. Modhia et al. ${ }^{26}$ report increasing readmission rates $(14.6 \%)$ within 2 years after surgery for spinal stenosis. Several authors highlight differences in the 30-day spinal surgery readmission rates, which depend on the complexity of the procedure ${ }^{8,21}$ and total operational time, ${ }^{21,24,30}$ so 
TABLE 3. Patient comorbidities and operative characteristics (after univariate analyses)

\begin{tabular}{|c|c|c|c|c|c|}
\hline \multirow[b]{2}{*}{ Characteristic } & \multicolumn{5}{|c|}{ Study } \\
\hline & $\begin{array}{l}\text { Deyo et al., } \\
2010\end{array}$ & $\begin{array}{c}\text { Jansson et al., } \\
2004\end{array}$ & $\begin{array}{c}\text { Kim et al., } \\
2014\end{array}$ & $\begin{array}{l}\text { Lovecchio et al., } \\
2014\end{array}$ & $\begin{array}{l}\text { Pugely et al., } \\
2014\end{array}$ \\
\hline \multicolumn{6}{|l|}{ Comorbidity } \\
\hline Alcohol abuse & 0 & 0 & NS & 0 & NS \\
\hline Anemia & 0 & 0 & $S$ & 0 & 0 \\
\hline Bleeding disorder & 0 & 0 & $S$ & NS & $S$ \\
\hline Chemotherapy & 0 & 0 & NS & 0 & NS \\
\hline $\mathrm{CHF}$ & 0 & 0 & NS & 0 & $S$ \\
\hline COPD & 0 & 0 & $S$ & NS & $S$ \\
\hline Diabetes & 0 & 0 & $S$ & $S$ & $S$ \\
\hline Dyspnea & 0 & 0 & $S$ & NS & NS \\
\hline Hypertension & 0 & 0 & $S$ & $S$ & $S$ \\
\hline Obesity & 0 & 0 & NS & 0 & 0 \\
\hline Prior op within 30 days & 0 & 0 & NS & NS & $S$ \\
\hline $\mathrm{PCl} /$ cardiac surgery & 0 & 0 & $S$ & $S$ & 0 \\
\hline Previous stroke/TIA & 0 & 0 & NS & $S$ & 0 \\
\hline Steroid use & 0 & 0 & $S$ & NS & $S$ \\
\hline Tobacco use & 0 & 0 & NS & NS & NS \\
\hline \multicolumn{6}{|l|}{ Operative } \\
\hline Emergency case & 0 & 0 & $S$ & NS & $S$ \\
\hline Total op time & 0 & 0 & $S$ & $S$ & $S$ \\
\hline Inpatient status & 0 & 0 & $S$ & 0 & 0 \\
\hline Outpatient status & 0 & 0 & 0 & 0 & $S$ \\
\hline
\end{tabular}

$\mathrm{CHF}=$ congestive heart failure $\mathrm{COPD}=$ chronic obstructive pulmonary disease $\mathrm{PCI}=$ percutaneous coronary intervention; $\mathrm{TIA}=$ transient ischemic attack.

future studies should include and standardize these variables in reporting their results.

Exploring the heterogeneity of reported results for lumbar disc surgery shows that Kim et al. ${ }^{21}$ reported unplanned readmission, whereas Jansson et al. ${ }^{19}$ did not distinguish between planned and unplanned readmission. In addition, the 2 studies were conducted in different countries (Sweden vs US) and covered different time periods (1987-1999 and 2011). Hence, it is very likely that surgical procedures, discharge criteria, and economic pressures differed significantly between the 2 groups.

We found that increasing age is consistently a risk factor for 30-day readmission. ${ }^{8,19,24,30}$ Age is also positively associated with mortality and complication rates in spine surgery, ${ }^{33}$ gastric bypass surgery, ${ }^{23}$ as well as many other surgical procedures, ${ }^{16,32,38}$ so it is not surprising that it is also associated with 30-day readmission. Older age has been associated with higher comorbidity status and higher ASA class, ${ }^{20}$ which alone have been reported as significant predictors of surgery outcomes. Jansson et al. ${ }^{19}$ found that age as a predictor for readmission varied depending on the age of the patient, with the lowest risk between ages 50 and 69 years and elevated risk for all ages below and above. With an aging demographic everywhere in the developed world, measures will need to be put in place to mitigate an overwhelming absolute number of readmissions in older patients.
The results regarding significance of sex as a predictor of readmission are inconclusive. Three studies found females to be more susceptible to return to the health care system within 30 days, $, 8,19,30$ while 2 studies found no significant difference. ${ }^{21,24}$ Other studies reporting on the neurosurgery of the spine show varying results as well. $., 6,25,31,32,36,38$

Three studies investigated ASA physical status classification and found it to be significantly elevated in the readmitted group..$^{21,24,30}$ Previously, the increasing ASA score has been connected to increased mortality and worse outcomes for several surgical procedures by Wolters et al. ${ }^{39}$

Comorbid illnesses were also an effective predictor of readmission. ${ }^{8,30}$ Presence of diabetes and hypertension prior to operation were consistently reported as risk factors. $^{21,24,30}$ This conclusion is not at all surprising, as studies usually have shown a significant association between diabetes or hypertension and poor postoperative improvement for various procedures. ${ }^{3,4,7,15,25,35}$ Diabetes and other comorbid conditions have also been noted as causes of a prolonged in-hospital stay, ${ }^{4}$ explaining why Jansson et al. ${ }^{19}$ and Pugely et al.$^{30}$ found extended LOS to be predictive of worse outcome.

Our review found that DVT, PE, and other venous complications are positively correlated with readmission after lumbar decompression. ${ }^{21}$ Similarly, after craniotomy, patients who developed venous complications or hydrocephalus were nearly 4 times and 58\%, respectively, more likely 
TABLE 4. Outcomes reported and their statistical contributions

\begin{tabular}{|c|c|c|c|c|c|}
\hline \multirow[b]{2}{*}{ Complication } & \multicolumn{5}{|c|}{ Study } \\
\hline & $\begin{array}{l}\text { Deyo et al., } \\
2010\end{array}$ & $\begin{array}{l}\text { Jansson et al., } \\
2004\end{array}$ & $\begin{array}{l}\text { Kim et al., } \\
2014\end{array}$ & $\begin{array}{l}\text { Lovecchio et al., } \\
2014\end{array}$ & $\begin{array}{l}\text { Pugely et al., } \\
2014\end{array}$ \\
\hline Overall & $X$ & 0 & 0 & $S$ & 0 \\
\hline Surgical and wound & $X$ & 0 & S & $S$ & $X$ \\
\hline Superficial SSI & 0 & 0 & S & 0 & 0 \\
\hline Deep/incisional SSI & 0 & 0 & S & 0 & 0 \\
\hline Organ/space SSI & 0 & 0 & S & 0 & 0 \\
\hline Wound dehiscence & 0 & 0 & $S$ & 0 & 0 \\
\hline Medical & 0 & 0 & $S$ & $S$ & 0 \\
\hline Pulmonary & $x$ & 0 & 0 & $S$ & 0 \\
\hline Pneumonia & 0 & 0 & $S$ & 0 & $x$ \\
\hline Unplanned reintubation & 0 & 0 & S & 0 & 0 \\
\hline Mechanical ventilation $>48 \mathrm{hrs}$ & 0 & 0 & NS & 0 & 0 \\
\hline Cardiac & $x$ & 0 & 0 & NS & $x$ \\
\hline Cardiac arrest & 0 & 0 & $S$ & 0 & $x$ \\
\hline Myocardial infarction & 0 & 0 & $S$ & 0 & $x$ \\
\hline Vascular & 0 & 0 & 0 & NS & $x$ \\
\hline PE & 0 & 0 & S & 0 & $x$ \\
\hline DVT & 0 & 0 & $S$ & 0 & $x$ \\
\hline Renal & 0 & 0 & 0 & NS & 0 \\
\hline Renal insufficiency & 0 & 0 & NS & 0 & 0 \\
\hline Acute renal failure & 0 & 0 & NS & 0 & 0 \\
\hline Neurocomplications & 0 & 0 & 0 & NS & $x$ \\
\hline Stroke & $x$ & 0 & S & 0 & $x$ \\
\hline Coma & 0 & 0 & $x$ & 0 & 0 \\
\hline Peripheral nerve injury & 0 & 0 & NS & 0 & 0 \\
\hline Genitourinary & 0 & 0 & 0 & 0 & $x$ \\
\hline UTI & 0 & 0 & $S$ & $S$ & $x$ \\
\hline Postop blood transfusion & 0 & 0 & S & $S$ & 0 \\
\hline Sepsis/septic shock & 0 & 0 & S & NS & $x$ \\
\hline Gastrointestinal & 0 & 0 & 0 & 0 & $X$ \\
\hline Respiratory & 0 & 0 & 0 & 0 & $x$ \\
\hline Spinal symptoms & 0 & $X$ & 0 & 0 & 0 \\
\hline Fluid and electrolytes & 0 & 0 & 0 & 0 & $x$ \\
\hline Trauma & 0 & 0 & 0 & 0 & $x$ \\
\hline Pain & 0 & 0 & 0 & 0 & $x$ \\
\hline Miscellaneous/other & 0 & 0 & 0 & 0 & $x$ \\
\hline Procedural & 0 & 0 & 0 & 0 & $x$ \\
\hline Reoperation & 0 & $x$ & $S$ & 0 & 0 \\
\hline Mortality & $X$ & 0 & 0 & NS & 0 \\
\hline
\end{tabular}

$\mathrm{SSI}=$ surgical site infection; $\mathrm{X}=$ included in the tested model but statistical significance was not reported or it was unclearly stated.

to return. ${ }^{25}$ Eisenring et al. ${ }^{9}$ as a result of their study, recommend using intraoperative leg elevation, intermittent pneumatic compression, early heparin administration, and low molecular weight heparin to reduce the risk for PE after skull base meningioma surgery. Pharmacological prophylaxis is recommended when surgically treating spinal cord injury; however, conclusive results are not available regarding degenerative spine surgery. ${ }^{34}$ These findings high- light the need for more effort to avoid these preventable events in ways that minimize the risk of bleeding within the central nervous system.

Anemia was more common among readmitted patients. ${ }^{21}$ This is not surprising since anemia has been shown to be a preoperative risk factor in the neurosurgery of the spine, associated with ileus in lumbar fusion, ${ }^{11}$ posterior lumbar spine surgery, ${ }^{14}$ and postoperative delirium 
after lumbar surgery. ${ }^{12}$ Intraoperative acute blood loss anemia also has been reported to relate to perioperative cardiac events following cervical and lumbar spine surgery. ${ }^{10,13}$ The results from our review indicate that receiving blood transfusion is protective against unplanned readmission. ${ }^{21}$ A potential explanation for this finding may be that although blood transfusion is often associated with higher rates of postoperative complications, morbidity, and mortality, ${ }^{1,18,21,29}$ it may be an effective way of dealing with those complications before leaving the hospital. That is, a patient who undergoes a large vascular spinal fusion procedure may be appropriately treated with blood transfusion. Our study suggests that it may be worse to let the patient's hemoglobin drop so low that they later return with a complication of anemia, such as new onset angina. Supporting this is Kim et al.'s ${ }^{21}$ finding that anemic patients are overrepresented in the readmitted population, but more research is clearly needed here.

Postoperative complications may be the most important predictor of 30-day readmission after neurosurgery of the spine ${ }^{8,21,24}$ We found that postoperative complications were predictive in multiple studies, and others found that they were a common risk factor for 30-day readmission across multiple specialties. $8,18,21,24,25,27,30,32$ Lovecchio et al. ${ }^{24}$ found that demographic factors, comorbid disease, and operative characteristics could not alone predict readmission rate, when complications remained as a covariate. Preoperative patient characteristics like diabetes, as shown above, increase the likelihood of complication, and these complications increase the risk of readmission. Identifying a common set of most frequent complications and their predisposing elements will allow for preventive measures, heightened surveillance, and anticipatory treatment in high risk patients like the elderly diabetic patient undergoing a lumbar spinal fusion.

In a univariate analysis of readmission after anterior cervical decompression and fusion, Lovecchio et al. ${ }^{24}$ showed the significant association between pulmonary complications, wound infection, UTI, blood transfusion, and readmission rates. In lumbar spinal decompression surgery, multivariate analyses highlight the importance of overall complications, pulmonary embolism, postoperative blood transfusion, and unplanned reoperation as independent risk factors for 30-day readmission. ${ }^{21}$ Patients undergoing unplanned reoperation were 56.91 times more likely to return within 30 days after lumbar decompression. ${ }^{21}$

\section{Limitations and Future Work}

Little research has been dedicated to readmission rates in neurosurgery and, more specifically, the neurosurgery of the spine as corroborated by the small number of studies we identified. The heterogeneity of studies that we identified precluded our ability to perform meta-analysis in this group of papers. Most papers were based on studies carried out in the United States and future work should encourage work from other jurisdictions, particularly those with different forms of health care financing. Future work should also be based on well-designed prospectively collected surveillance data on very large groups of patients. In this respect, organized neurosurgery through groups like the NeuroPoint Alliance and others may play a central role in moving the field forward.

\section{Conclusions}

Our review highlights the central importance of factors associated with the patient, specifically age and preoperational comorbidities like diabetes, in predicting 30-day readmission. It also emphasizes the importance of surgical complication avoidance, including avoiding heavy blood loss during surgery in preventing readmission. Fortunately, neurosurgeons can be proactive in designing strategies for prevention of readmission relating to both of these overarching factors.

With regards to the patient-centered factors, processes should be set in place in surgical programs to identify and manage elderly patients with several comorbidities and limitations such as those associated with mobility and subsequent DVT and PE like limb paresis. Although as surgeons we often see these factors, many of our surgical programs do not have protocoled plans to manage these patients before, during, and after surgery. These programs can easily be built into standardized protocols that nurse practitioners and other health care professionals in the neurosurgical team can implement. For instance, protocols around fall prevention and the removal of nonessential urinary catheters and indwelling central venous catheters to reduce nosocomial infections ${ }^{32}$ should be instituted widely. Education focusing on the benefits of practices like early mobility and rehabilitation should be delivered by trained professionals in a standardized fashion to all these patients and their families/caregivers preoperatively, in hospital, and after discharge. Programs should have wellestablished protocols around the prevention of PE using measures like sequential compression devices and biochemical prophylaxis.

Complication avoidance during neurosurgery is directly within the purview of the neurosurgeon and the neurosurgical team. This goes beyond good surgical technique, which is a sine qua non. Minimizing blood loss and damage of eloquent tissues are clearly 2 overarching themes in good surgical technique. Detailed and procedure-specific checklists that all team members complete and are redone whenever team transitions occur during the surgical or postsurgical process (e.g., intensive care unit or at discharge from hospital) are critical. Every team should also conduct regular multidisciplinary audits in a nonaccusatory and nonpunitive fashion that sincerely strive to identify gaps in the care pathway and network that lead to near misses, errors, and complications in the operating room. An equally open collaborative atmosphere is required to institute solutions to these problems. These solutions should be shared with the wider neurosurgical community so that efficiency is built into these quality and best practice improvements. A systems approach to this end has been well established in the airline industry and increasingly in the practice of anesthesia. ${ }^{2,28}$ Neurosurgery would fare well to institutionalize these sorts of processes globally. Finally, neurosurgery needs to prioritize quality and best practice research to move the field forward. Studies assessing critical quality and best practice issues, such 
as when to implement prophylactic anticoagulation during and after neurosurgery, must be a priority.

\section{Acknowledgments}

We would like to thank Dr. Sanjit K. Bhogal and David Lightfoot for assisting with the conception and design of the database search and organization.

\section{References}

1. Al-Refaie WB, Parsons HM, Markin A, Abrams J, Habermann EB: Blood transfusion and cancer surgery outcomes: a continued reason for concern. Surgery 152:344-354, 2012

2. Anesthesia Patient Safety Foundation: About APSF: Foundation History. Indianapolis: Anesthesia Patient Safety Foundation, 2010 (http://www.apsf.org/about_history.php) [Accessed July 16, 2015]

3. Appaduray SP, Lo P: Effects of diabetes and smoking on lumbar spinal surgery outcomes. J Clin Neurosci 20:17131717, 2013

4. Bucerius J, Gummert JF, Walther T, Doll N, Falk V, Onnasch JF, et al: Impact of diabetes mellitus on cardiac surgery outcome. Thorac Cardiovasc Surg 51:11-16, 2003

5. Buchanan CC, Hernandez EA, Anderson JM, Dye JA, Leung M, Buxey F, et al: Analysis of 30-day readmissions among neurosurgical patients: surgical complication avoidance as key to quality improvement. J Neurosurg 121:170-175, 2014

6. Chern JJ, Bookland M, Tejedor-Sojo J, Riley J, Shoja MM, Tubbs RS, et al: Return to system within 30 days of discharge following pediatric shunt surgery. J Neurosurg Pediatr 13:525-531, 2014

7. Clough RA, Leavitt BJ, Morton JR, Plume SK, Hernandez F, Nugent W, et al: The effect of comorbid illness on mortality outcomes in cardiac surgery. Arch Surg 137:428-433, 2002

8. Deyo RA, Mirza SK, Martin BI, Kreuter W, Goodman DC, Jarvik JG: Trends, major medical complications, and charges associated with surgery for lumbar spinal stenosis in older adults. JAMA 303:1259-1265, 2010

9. Eisenring CV, Neidert MC, Sabanés Bové D, Held L, Sarnthein J, Krayenbühl N: Reduction of thromboembolic events in meningioma surgery: a cohort study of 724 consecutive patients. PLoS One 8:e79170, 2013

10. Fineberg SJ, Ahmadinia K, Patel AA, Oglesby M, Singh K: Incidence and mortality of cardiac events in lumbar spine surgery. Spine (Phila Pa 1976) 38:1422-1429, 2013

11. Fineberg SJ, Nandyala SV, Kurd MF, Marquez-Lara A, Noureldin M, Sankaranarayanan S, et al: Incidence and risk factors for postoperative ileus following anterior, posterior, and circumferential lumbar fusion. Spine J 14:1680-1685, 2014

12. Fineberg SJ, Nandyala SV, Marquez-Lara A, Oglesby M, Patel AA, Singh K: Incidence and risk factors for postoperative delirium after lumbar spine surgery. Spine (Phila Pa 1976) 38:1790-1796, 2013

13. Fineberg SJ, Oglesby M, Patel AA, Singh K: Incidence and mortality of perioperative cardiac events in cervical spine surgery. Spine (Phila Pa 1976) 38:1268-1274, 2013

14. Gruskay JA, Fu MC, Bohl DD, Webb ML, Grauer JN: Factors affecting length of stay after elective posterior lumbar spine surgery: a multivariate analysis. Spine J 15:1188-1195, 2015

15. Guzman JZ, Iatridis JC, Skovrlj B, Cutler HS, Hecht AC, Qureshi SA, et al: Outcomes and complications of diabetes mellitus on patients undergoing degenerative lumbar spine surgery. Spine (Phila Pa 1976) 39:1596-1604, 2014

16. Hamel MB, Henderson WG, Khuri SF, Daley J: Surgical outcomes for patients aged 80 and older: morbidity and mortality from major noncardiac surgery. J Am Geriatr Soc 53:424-429, 2005
17. Health Canada: Healthy Canadians-A Federal Report on Comparable Health Indicators 2012. Ottawa: Health Canada, 2013, pp 43-46

18. Hopewell S, Omar O, Hyde C, Yu LM, Doree C, Murphy MF: A systematic review of the effect of red blood cell transfusion on mortality: evidence from large-scale observational studies published between 2006 and 2010. BMJ Open 3:e002154, 2013

19. Jansson KA, Németh G, Granath F, Blomqvist P: Surgery for herniation of a lumbar disc in Sweden between 1987 and 1999. An analysis of 27,576 operations. J Bone Joint Surg Br 86:841-847, 2004

20. Khan MR, Bari H, Zafar SN, Raza SA: Impact of age on outcome after colorectal cancer surgery in the elderly - a developing country perspective. BMC Surg 11:17, 2011

21. Kim BD, Smith TR, Lim S, Cybulski GR, Kim JYS: Predictors of unplanned readmission in patients undergoing lumbar decompression: multi-institutional analysis of 7016 patients. J Neurosurg Spine 20:606-616, 2014

22. Liberati A, Altman DG, Tetzlaff J, Mulrow C, Gøtzsche PC, Ioannidis JPA, et al: The PRISMA statement for reporting systematic reviews and meta-analyses of studies that evaluate health care interventions: explanation and elaboration. PLoS Med 6:e1000100, 2009

23. Livingston EH, Huerta S, Arthur D, Lee S, De Shields S, Heber D: Male gender is a predictor of morbidity and age a predictor of mortality for patients undergoing gastric bypass surgery. Ann Surg 236:576-582, 2002

24. Lovecchio F, Hsu WK, Smith TR, Cybulski G, Kim B, Kim JYS: Predictors of thirty-day readmission after anterior cervical fusion. Spine (Phila Pa 1976) 39:127-133, 2014

25. Marcus LP, McCutcheon BA, Noorbakhsh A, Parina RP, Gonda DD, Chen C, et al: Incidence and predictors of 30-day readmission for patients discharged home after craniotomy for malignant supratentorial tumors in California (19952010). J Neurosurg 120:1201-1211, 2014

26. Modhia U, Takemoto S, Braid-Forbes MJ, Weber M, Berven SH: Readmission rates after decompression surgery in patients with lumbar spinal stenosis among Medicare beneficiaries. Spine (Phila Pa 1976) 38:591-596, 2013

27. Nuño M, Ly D, Ortega A, Sarmiento JM, Mukherjee D, Black KL, et al: Does 30-day readmission affect long-term outcome among glioblastoma patients? Neurosurgery 74:196-205, 2014

28. O'Daniel M, Rosenstein AH: Professional communication and team collaboration, in Patient Safety and Quality: An Evidence-Based Handbook for Nurses. Rockville, MD: Agency for Healthcare Research and Quality, 2008, Vol 2, pp 271-284

29. O'Keeffe SD, Davenport DL, Minion DJ, Sorial EE, Endean ED, Xenos ES: Blood transfusion is associated with increased morbidity and mortality after lower extremity revascularization. J Vasc Surg 51:616-621, 621.e1-621.e3, 2010

30. Pugely AJ, Martin CT, Gao Y, Mendoza-Lattes S: Causes and risk factors for 30-day unplanned readmissions after lumbar spine surgery. Spine (Phila Pa 1976) 39:761-768, 2014

31. Rambachan A, Smith TR, Saha S, Eskandari MK, Bendok BR, Kim JYS: Reasons for readmission after carotid endarterectomy. World Neurosurg 82:e771-e776, 2014

32. Shah MN, Stoev IT, Sanford DE, Gao F, Santiago P, Jaques DP, et al: Are readmission rates on a neurosurgical service indicators of quality of care? J Neurosurg 119:1043-1049, 2013

33. Smith JS, Saulle D, Chen CJ, Lenke LG, Polly DW Jr, Kasliwal MK, et al: Rates and causes of mortality associated with spine surgery based on 108,419 procedures: a review of the Scoliosis Research Society Morbidity and Mortality Database. Spine (Phila Pa 1976) 37:1975-1982, 2012

34. Strom RG, Frempong-Boadu AK: Low-molecular-weight 
heparin prophylaxis 24 to 36 hours after degenerative spine surgery: risk of hemorrhage and venous thromboembolism. Spine (Phila Pa 1976) 38:E1498-E1502, 2013

35. Takahashi S, Suzuki A, Toyoda H, Terai H, Dohzono S, Yamada K, et al: Characteristics of diabetes associated with poor improvements in clinical outcomes after lumbar spine surgery. Spine (Phila Pa 1976) 38:516-522, 2013

36. Vaziri S, Cox JB, Friedman WA: Readmissions in neurosurgery: a qualitative inquiry. World Neurosurg 82:376-379, 2014

37. von Elm E, Altman DG, Egger M, Pocock SJ, Gøtzsche PC, Vandenbroucke JP: The Strengthening the Reporting of Observational Studies in Epidemiology (STROBE) statement: guidelines for reporting observational studies. J Clin Epidemiol 61:344-349, 2008

38. Wang MC, Shivakoti M, Sparapani RA, Guo C, Laud PW, Nattinger AB: Thirty-day readmissions after elective spine surgery for degenerative conditions among US Medicare beneficiaries. Spine J 12:902-911, 2012

39. Wolters U, Wolf T, Stützer H, Schröder T: ASA classification and perioperative variables as predictors of postoperative outcome. Br J Anaesth 77:217-222, 1996

\section{Disclosure}

The authors report no conflict of interest concerning the materials or methods used in this study or the findings specified in this paper.

\section{Author Contributions}

Conception and design: all authors. Acquisition of data: Pshonyak, Lee. Analysis and interpretation of data: all authors. Drafting the article: all authors. Critically revising the article: all authors. Reviewed submitted version of manuscript: all authors. Approved the final version of the manuscript on behalf of all authors: Cusimano. Administrative/technical/material support: Cusimano, Ilie. Study supervision: Ilie.

\section{Correspondence}

Michael D. Cusimano, St. Michael's Hospital, Division of Neurosurgery, 30 Bond St., Toronto, ON M5B 1W8, Canada. email: injuryprevention@smh.ca. 